\title{
Reply to "Utility of Intraoperative Margin Assessment by Frozen Section in Gastric Cancer"
}

\author{
Koki Nakanishi, MD ${ }^{1}$, Shinji Morita, $\mathrm{MD}^{1}$, Hirokazu Taniguchi, $\mathrm{MD}^{2}$, Sho Otsuki, $\mathrm{MD}^{1}$, \\ Takeo Fukagawa, $\mathrm{MD}^{1}$, and Hitoshi Katai, $\mathrm{MD}^{1}$ \\ ${ }^{1}$ Department of Gastric Surgery, National Cancer Center Hospital, Tokyo, Japan; ${ }^{2}$ Pathology and Clinical Laboratory \\ Division, National Cancer Center Hospital, Tokyo, Japan
}

Dear Editor,

We thank Dr. Squires for his letter to the editor entitled "Utility of Intraoperative Margin Assessment by Frozen Section in Gastric Cancer," which stimulates discussion around an important clinical question.

Although we revealed that intraoperative margin assessment by frozen section (FS) was a highly accurate examination, we could not show the oncologic utility of achieving macroscopic negative (R0) on permanent section (PS) using intraoperative margin assessment by FS. ${ }^{1}$ Squires et al. reported that margin re-resection for FS-R1 was not associated with improved relapse-free survival and overall survival compared with the PS-R1 group. ${ }^{2}$ In addition, several studies have reported that reresection for R1 margin in the setting of pT3-4 or pN2-3 gastric cancer did not confer any oncologic benefit, because the main recurrence pattern in patients with positive margin was distant rather than locoregional. ${ }^{3,4}$ However, these studies cannot sufficiently eliminate the confounding effect of the difference in postoperative treatment that affects survival outcome. Usually, patients with positive margin will undergo more intensive postoperative treatment compared with those with R0 margin, such as systemic chemotherapy or chemoradiotherapy. In Squires' study, the frequency of receiving postoperative treatment tended to be high in patients with positive margin, ${ }^{2}$ and no postoperative treatment data were provided by Bickenbach et al. ${ }^{3}$ and Sun et al. ${ }^{4}$ Margin re-resection for FS-R1 may be beneficial for patients in term of avoiding such additional treatment, if oncologic outcome is better or equal.

Another concern is the survival difference between the FS-R1 converted to PS-R0 group and the FS-R0 group. However, there are clinicopathological differences between

(C) Society of Surgical Oncology 2019

First Received: 10 June 2019;

Published Online: 23 July 2019

S. Morita, MD

e-mail: smorita@ncc.go.jp these groups, with larger tumor size and $\mathrm{T} 4$ tumor being independent factors associated with FS-R1 in our study, ${ }^{1}$ similar to other studies. ${ }^{2,4}$ An analysis that balances these variables strictly, e.g., propensity score matching, is desirable to solve this question.

Finally, further analysis is needed to investigate the utility of re-resection to achieve R0 after FS-R1. It would be difficult to perform such clinical trials which determine this oncologic outcome from an ethical aspect. In fact, a single-institution study had a small number of patients, and geographical differences are observed in postoperative treatment strategies for gastric cancer patients. To overcome these limitations, reliable and high-quality evidence based on accumulated data are required.

\section{REFERENCES}

1. Nakanishi K, Morita S, Taniguchi H, Otsuki S, Fukagawa T, Katai H. Diagnostic accuracy and usefulness of intraoperative margin assessment by frozen section in gastric cancer. Ann Surg Oncol. 2019;26(6):1787-94.

2. Squires MH, 3rd, Kooby DA, Pawlik TM, et al. Utility of the proximal margin frozen section for resection of gastric adenocarcinoma: a 7-institution study of the US Gastric Cancer Collaborative. Ann Surg Oncol. 2014;21(13):4202-10.

3. Bickenbach KA, Gonen M, Strong V, Brennan MF, Coit DG. Association of positive transection margins with gastric cancer survival and local recurrence. Ann Surg Oncol. 2013;20(8):2663-8.

4. Sun Z, Li DM, Wang ZN, et al. Prognostic significance of microscopic positive margins for gastric cancer patients with potentially curative resection. Ann Surg Oncol. 2009;16(11):3028-37.

Publisher's Note Springer Nature remains neutral with regard to jurisdictional claims in published maps and institutional affiliations. 